\title{
Fragmented type II burst emission during CME liftoff
}

\author{
Silja Pohjolainen ${ }^{1}$, Jens Pomoell ${ }^{2}$ and Rami Vainio ${ }^{2}$ \\ ${ }^{1}$ Department of Physics and Astronomy, University of Turku, \\ Tuorla Observatory, 21500 Piikkiö, Finland \\ email: silpoh@utu.fi \\ ${ }^{2}$ Department of Physics, University of Helsinki, \\ PO Box 64, 00014 University of Helsinki, Finland \\ email: jens.pomoell@helsinki.fi, rami.vainio@helsinki.fi
}

\begin{abstract}
We have performed multiwavelength analysis on an event with a metric type II burst, which appeared first as fragmented emission lanes in the radio dynamic spectrum. The start frequency was unusually high. Since type II bursts are thought to be signatures of propagating shock waves, it is of interest to know how the shocks, and the type II bursts, are formed. This radio event was associated with a flare and a coronal mass ejection (CME), and we investigate their connection. Observations suggested that a propagating shock was formed due to the erupting structures, and the observed radio emission reflects the high densities in active region loops. We then utilised numerical MHD simulations, to study the shock structure induced by an erupting CME, in a model corona including dense loops. Our simulations show that the fragmented part of the type II burst can be formed when a coronal shock driven by a CME passes through a system of dense loops overlying an active region. To produce fragmented emission, the conditions for plasma emission have to be more favourable inside the loop than in the inter-loop area. The obvious hypothesis, consistent with our simulation model, is that the shock strength decreases significantly in the space between the denser loops. Outside the active region, the type II burst dies out when the changing geometry no longer favours the electron shock-acceleration.
\end{abstract}

Keywords. Sun: coronal mass ejections (CMEs); flares; radio radiation, shock waves, plasmas

\section{Introduction}

Radio type II bursts are observed in association with flares and coronal mass ejections (CMEs). Metric type II bursts can be observed in dynamic radio spectra as slowly drifting emission lanes, with drift rates approximately at $0.1-1.0 \mathrm{MHz} \mathrm{s}^{-1}$ (Nelson \& Melrose 1985). The start frequency of metric type II bursts is usually at about $100-200 \mathrm{MHz}$. The mechanism behind the bursts is generally assumed to be a propagating shock which creates electron beams that excite Langmuir waves, which in turn convert into radio waves at the local plasma frequency and its harmonics.

The exact relationship between solar flares, shocks, and coronal mass ejections is still not well understood, and it is of interest to know how shocks are initiated and under which conditions radio type II bursts can be excited. As shocks can be formed in various ways, it is not evident that all solar radio type II bursts are formed in the same way. In particular, how are untypical, fragmented type II bursts that start at very high frequencies created? Are there differences in shock acceleration or in the surrounding medium that can explain the differences to the "typical" metric type IIs? 


\section{Analysis}

We have analysed in detail one metric type II burst that occurred on 13 May 2001. The burst started at an unusually high frequency and proceeded showing fragmented and curved emission bands, which were visible at the fundamental and second harmonic plasma frequencies (Fig. 1). The first "fragment" showed emission between 500 and 420 $\mathrm{MHz}$, which correspond to densities in the range of $2-3 \times 10^{9} \mathrm{~cm}^{-3}$. The second fragment near 03:02 UT gives densities $1-2 \times 10^{9} \mathrm{~cm}^{-3}(400-310 \mathrm{MHz})$, and the third near 03:03 UT gives densities $2-6 \times 10^{8} \mathrm{~cm}^{-3}(220-130 \mathrm{MHz})$. These values indicate that the source regions were dense, similar to active region loops. The frequency drifts (fundamental emission) within the fragments were between 1.8 and $4.3 \mathrm{MHz} \mathrm{s}^{-1}$.

The flare was well-observed in X-rays (Yohkoh) and EUV (TRACE). The images show a filament eruption, where most of the material is moving toward the Southeast. The outermost front of the filament moves with a projected speed of about $380 \mathrm{~km} \mathrm{~s}^{-1}$, but there is also a separate 'blob' that moves more to the Southwest with a projected speed of $450 \mathrm{~km} \mathrm{~s}^{-1}$. White-light observations from SOHO LASCO revealed a CME front moving toward the South at a speed of $430 \mathrm{~km} \mathrm{~s}^{-1}$. A soft X-ray loop system was observed to move also southward, at a projected speed of about $650 \mathrm{~km} \mathrm{~s}^{-1}$. Using the Yohkoh SXT filter ratio method (see, for example, McTiernan et al. 1993), we found that the soft X-ray loop densities agreed with the plasma densities of the fragmented metric type II burst (Pohjolainen et al. 2008).

\section{Simulations}

We then utilised numerical MHD simulations to study the shock structure induced by a CME, in a model corona including dense loops. The details of the model are presented in Pomoell et al. (2008). We considered three different runs with slightly different parameters. The plot in Fig. 1 shows the radio emission produced by a shock, assuming that the emission is produced immediately in front of the CME leading edge shock. The emission lanes show a frequency drop in accordance with the exponentially decreasing density of the ambient corona. However, when the radio-emitting shock propagates in a dense loop, the frequency is higher, and drops quickly when the shock exits the loop (near $\mathrm{t}=200 \mathrm{~s}$ ). Additionally, we indicate the compression ratio of the shock by the size
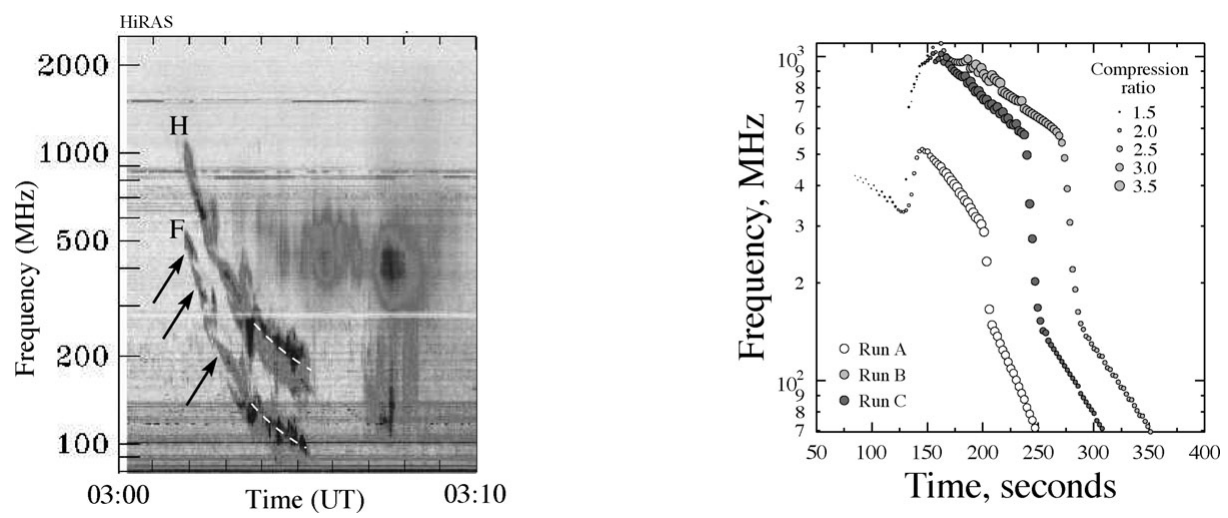

Figure 1. Left: HiRAS dynamic spectrum from the $25-2500 \mathrm{MHz}$ frequency range, at 03:00 - 03:10 UT on 13 May 2001. 'F' notes emission at the fundamental and ' $H$ ' at the second harmonic plasma frequency. Arrows point to the fragmented emission bands and dashed white lines outline the later-appearing "regular" type II burst lanes. Right: Radio track of three different simulation runs. The size of the marker indicates the compression ratio of the shock. 


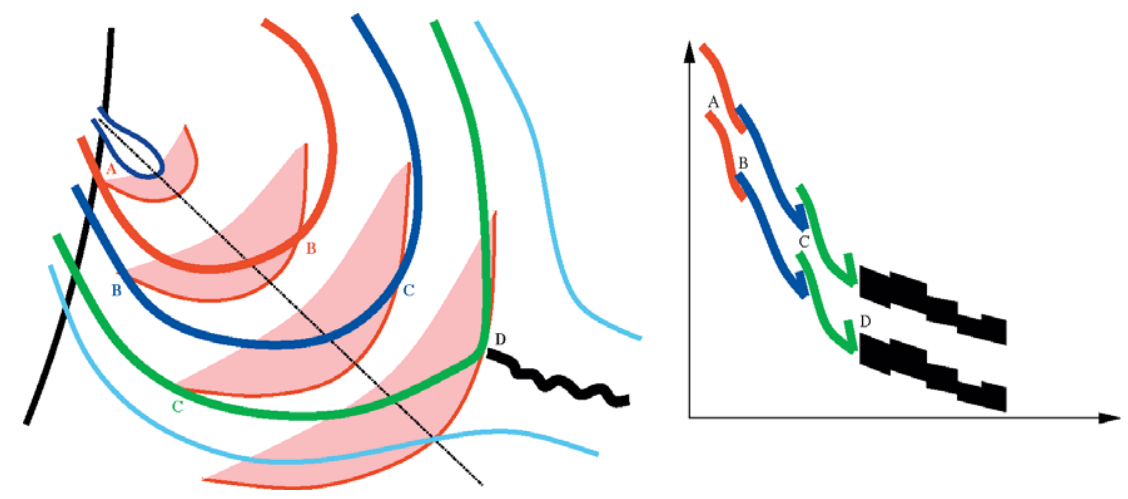

Figure 2. A possible scenario for a fragmented type II burst

of the marker. In all three cases, the shock is strong while it propagates in the loop, with the compression ratio remaining between 3 and 3.5.

The dynamics of the eruption is as follows: As the flux rope starts to rise, a perturbation is formed around the flux rope. Due to the gradient in the Alfvén speed and the increasing speed of the flux rope, the wave steepens to a shock ahead of the flux rope. However, the strength of the shock remains weak in the area below the loop. When the shock reaches the dense loop, it strengthens and slows down quickly due to the low Alfvén speed in the loop. The erupting filament continues to push the loop structure ahead of it, acting as the driver of the shock. Thus, the speed of the shock is roughly that of the displaced loop structure when propagating in the region of low Alfvén speed. As the filament decelerates, the displaced loop and shock escape from the filament. When reaching the region of higher Alfvén speed, the speed of the shock increases with the increasing Alfvén speed, and the shock escapes from the propagating loop structure.

\section{Model}

A possible scenario for the fragmented type II burst can be the following (Fig. 2): An erupting filament drives a strong shock wave through a system of overlying loops. Among these loops, a few are more favourable sites for plasma emission than others, e.g., because of their higher density or suprathermal electron content. These loops are "lit" in radio as the shock traverses them, producing the fragmented part of the radio burst. The regular part of the burst may then come from the part of the active region above the loop system, e.g., from an overlying current sheet. (See, e.g., Mancuso \& Abbo 2004, for a similar geometric model involving the interaction of a shock and a current sheet.) Finally, the burst dies out or even stops abruptly once the shock propagates out of the region completely.

\section{References}

Mancuso, S. \& Abbo, L. 2004, A\&\&A, 415, L17

McTiernan, J.M., Kane, S.R., Loran, J.M., et al. 1993, ApJ, 416, L91

Nelson, G. J. \& Melrose, D. B., 1985, in Solar Radiophysics, D. J. McLean and N. R. Labrum (eds.), Cambridge Univ. Press, 333

Pohjolainen, S., Pomoell, J., \& Vainio, R. 2008, A\&A, in press

Pomoell, J., Vainio, R., \& Kissmann, R. 2008, Solar Phys., in press 